\title{
Chiral spiral induced by a strong magnetic field
}

\author{
Hiroaki Abuki ${ }^{1, \star}$ \\ ${ }^{1}$ Department of Education, Aichi University of Education, 1 Hirosawa, Igaya-cho, Kariya 448-8542, Japan
}

\begin{abstract}
We study the modification of the chiral phase structure of QCD due to an external magnetic field. We first demonstrate how the effect of magnetic field can systematically be incorporated into a generalized Ginzburg-Landau framework. We then analyze the phase structure in the vicinity of the chiral critical point. In the chiral limit, the effect is found to be so drastic that it brings a "continent" of chiral spiral in the phase diagram, by which the chiral tricritical point is totally washed out. This is the case no matter how small the intensity of magnetic field is. On the other hand, the current quark mass protects the chiral critical point from a weak magnetic field. However, the critical point will eventually be covered by the chiral spiral phase as the magnetic field grows.
\end{abstract}

\section{Introduction}

There has recently been a growing interest on possible crystal structures formed by the chiral condensates in QCD at finite density [1-3]. On the other hand, the effect of magnetic field on QCD has also been the subject of intensive studies. Phenomenologically, exploring possible forms of strongly interacting matter under the magnetic field is relevant to the physics of magnetars; the compact stellar objects known to have a strong magnetic field, $B \sim 10^{10} \mathrm{~T}$ [4]. It also brings some impacts on the physics of heavy ion collisions which could produce a huge magnetic field $B \sim 10^{14} \mathrm{~T}$ in the very early stage of noncentral collisions [5]. There have been made a lot of theoretical approaches to the effects of magnetic field on QCD phase diagram. These include computations based on phenomenological models [6-8] as well as the lattice QCD simulations $[9,10]$. The former approaches predict the "magnetic catalysis", while the latter gives the opposite effect known as the "inverse magnetic catalysis". The mechanism for the magnetic catalysis is rather transparent, but that for the inverse one remains still a matter of active debates [11-14].

In this article, we report our recent study on the effect of strong magnetic fields on the chiral phases with a particular focus put on how it modifies the phase structure in the vicinity of the critical point. Several studies are already devoted on how the magnetic field affects the critical points $[15,16]$. For example, a new critical point is suggested to appear in the presence of a strong magnetic field [17]. There are also some work related to inhomogeneous phases under a strong magnetic field; these include the widening of the phase for solitonic modulation [18], the hybrid chiral condensate where the space varying phase is attached to the Real-Kink Crystal (RKC) [19]. The effect of current quark mass is also studied in [20]; it was shown that the chiral spiral aka the dual chiral density wave

^e-mail: abuki@auecc.aichi-edu.ac.jp 
(DCDW) survives as the "massive dual chiral density wave" where the complex phase of condensate gets skewed from a linear function of space coordinate, say, $z$.

We here concentrate on the neighborhood of the critical point. We first show that in this case it is possible to derive systematically the generalized Ginzburg-Landau (gGL) action without specifying any details about the spatial form of the chiral condensate. We derive this functional up to the first nontrivial order in the current quark mass $h$ and the magnetic field $B$. Based on the derived functional, we analyze the phases near the critical point. It turns out that these two ingredients have competing effects on inhomogeneous phases. In particular, the condensate accompanied by the complex phase, the chiral spiral, is found to be favored by the magnetic field [19], and accordingly the phase diagram gets drastically changed once the effect of magnetic field prevails.

\section{Deriving generalized Ginzburg-Landau action}

The generalized Ginzburg-Landau (gGL) action density in the absence of the external magnetic field can be derived in the same way as described in [21,22]. The quark loop contribution to the effective action can be expanded in the power of the quark self-energy $\Sigma(\mathbf{x})=m_{q}+\sigma(\mathbf{x})+i \gamma_{5} \boldsymbol{\tau} \cdot \boldsymbol{\pi}(\mathbf{x})$ as

$$
\delta S_{\text {eff }}=\frac{T}{2} \sum_{n} \int d \mathbf{x} \int d \mathbf{y t r}\left[S\left(i \omega_{n}, \mathbf{x}-\mathbf{y}\right) \Sigma(\mathbf{y}) S\left(i \omega_{n}, \mathbf{y}-\mathbf{x}\right) \Sigma(\mathbf{x})\right]+O\left(\Sigma^{4}\right) .
$$

Here $S\left(i \omega_{n}, \mathbf{x}\right)=-\int d \mathbf{p} e^{i \mathbf{p} \cdot \mathbf{x}} \frac{i \omega_{n} \gamma_{0}-\mathbf{p} \cdot \gamma}{\omega_{n}^{2}+\mathbf{p}^{2}}$ is the quark propagator with $\omega_{n}=\pi T(2 n-1)$ being the Matsubara frequency. Expressing $\Sigma(\mathbf{y})=\Sigma(\mathbf{x})+\sum_{i=1}^{\infty} \frac{1}{i !}[(\mathbf{y}-\mathbf{x}) \cdot \nabla \Sigma(\mathbf{x})]^{i}$, we can perform a systematic derivative expansion of the effective action. Writing the action with the gGL action density $\omega$ as $S_{\text {eff }}=\int d \mathbf{x} \omega(\mathbf{x})$, the result is found up to the sixth order in $\sigma, \pi_{a}(a=1,2,3)$ and $\boldsymbol{\nabla} \equiv \boldsymbol{\partial}_{\mathbf{x}}$ as

$$
\begin{aligned}
\omega(\mathbf{x})= & \delta_{m} \omega(\mathbf{x})+\frac{\alpha_{2}}{2} \phi^{2}+\frac{\alpha_{4}}{4}\left(\phi^{4}+(\boldsymbol{\nabla} \phi)^{2}\right) \\
& +\frac{\alpha_{6}}{6}\left(\phi^{6}+3\left[\phi^{2}(\boldsymbol{\nabla} \phi)^{2}-(\phi \cdot \nabla \phi)^{2}\right]+5(\phi \cdot \nabla \phi)^{2}+\frac{1}{2}(\Delta \phi)^{2}\right),
\end{aligned}
$$

where we have switched to the chiral four-vector notation $\phi=(\sigma, \pi) . \quad \delta_{m} \omega(\mathbf{x})=-h \sigma$, which is the explicit symmetry breaking term associated with the current quark mass $m_{q}$, hereafter, called "hterm". $h$ and $\alpha_{n}(n=2,4,6)$, are the Ginzburg-Landau (GL) couplings which depend on temperature $T$ and chemical potential $\mu$. $h$ is proportional to $m_{q}$, and its explicit form is

$$
h=m_{q}\left(4 N_{c} N_{f} T \sum_{n} \int \frac{d \mathbf{p}}{(2 \pi)^{3}} \frac{1}{\left(\omega_{n}-i \mu\right)^{2}+\mathbf{p}^{2}}\right) .
$$

$N_{c(f)}$ is the number of color (flavor). The integral is divergent in ultra-violet and needs some regularization scheme to be evaluated. In the spirit of the GL approach, we simply take $h$ as a parameter characterizing the explicit symmetry breaking. Similarly the expressions for $\alpha_{n}$ can be found. There is an extra tree-level counter-contribution to $\alpha_{2}$ for the case of the standard NJL model [2]:

$$
\alpha_{2 i}=\frac{\delta_{i, 1}}{2 G}+(-1)^{i} 4 N_{c} N_{f} T \sum_{n} \int \frac{d \mathbf{p}}{(2 \pi)^{3}} \frac{1}{\left(\left(\omega_{n}-i \mu\right)^{2}+\mathbf{p}^{2}\right)^{i}},
$$

where $G$ is the NJL coupling constant for a four-quark (chiral-invariant) interaction. The integral is divergent for $\alpha_{2}$ and $\alpha_{4}$. These parameters are zero at the tricritical point $\left(\mu_{\mathrm{TCP}}, T_{\mathrm{TCP}}\right)$ which is expected to show up in the phase diagram in the chiral limit $m_{q}=0(h=0)$. 
Now we come to consider the effect of an external magnetic field. There is a direct effect on the quark propagator, whereas that on gluon sector is somehow indirect. It is easy to expand quark propagator in the power of magnetic field along with the line described in [23]:

$$
S\left(i \omega_{n}, \mathbf{p}\right) \rightarrow S\left(i \omega_{n}, \mathbf{p}\right)+\left(Q B_{i}\right) \frac{\not \phi_{\|}+\not \mu}{\left[\left(i \omega_{n}+\mu\right)^{2}-\mathbf{p}^{2}\right]^{2}} \frac{i \epsilon_{i j k} \gamma^{j} \gamma^{k}}{2}+O(Q \mathbf{B})^{2},
$$

where we have used the four vector notation $p_{\|}^{\mu}=\left(i \omega_{n}+\mu, \mathbf{p}_{\|}\right)$with $\mathbf{p}_{\|}=(\mathbf{p} \cdot \boldsymbol{B}) \boldsymbol{B} /|\boldsymbol{B}|^{2}$ being the parallel component of momentum. $Q=$ diag. $(2 e / 3,-e / 3)$ is the electric charge matrix in the flavor space. The first nontrivial term depending on $\boldsymbol{B}$ comes from the second order term in Eq. (1). Plugging Eq. (3) into the integrand of Eq. (1), and extracting the term linear in $\boldsymbol{B}$, we have

$$
\delta \omega_{B}(\mathbf{x})=\frac{T}{2} \sum_{n} \int \frac{d \mathbf{p}}{(2 \pi)^{3}} \frac{1}{\left[\left(i \omega_{n}+\mu\right)^{2}-\mathbf{p}^{2}\right]^{8}} \operatorname{tr}\left[\not p_{\|}\left(B_{i} \epsilon_{i j k} \gamma^{j} \gamma^{k}\right) Q \Sigma(\mathbf{x}) \not p \gamma^{l} \not p\left(\partial_{l} \Sigma(\mathbf{x})\right)\right] .
$$

Performing the traces over the Dirac, color and flavor spaces, we have

$$
\delta \omega_{B}(\mathbf{x})=e \boldsymbol{B} \cdot\left(\pi_{3} \boldsymbol{\nabla} \sigma-\sigma \nabla \pi_{3}\right) N_{c} T \sum_{n} \int \frac{d \mathbf{p}}{(2 \pi)^{3}} \frac{4\left(i \omega_{n}+\mu\right)}{\left[\left(i \omega_{n}+\mu\right)^{2}-\mathbf{p}^{2}\right]^{3}} .
$$

The Matsubara sum and integral over $\mathbf{p}$ can be done analytically, and the result will be expressed by the generalized zeta function. However, its explicit form is of no importance here. Instead of writing the result, we only note that the result can be written with the derivative of $\alpha_{4}$ with respect to $\mu$.

$$
\delta \omega_{B}(\mathbf{x})=-\frac{1}{4 N_{f}} \frac{\partial \alpha_{4}}{\partial \mu} e \boldsymbol{B} \cdot\left(\pi_{3} \boldsymbol{\nabla} \sigma-\sigma \nabla \pi_{3}\right) \equiv-\boldsymbol{b} \cdot\left(\sigma \nabla \pi_{3}-\pi_{3} \boldsymbol{\nabla} \sigma\right) .
$$

We introduced a new GL coupling $\boldsymbol{b}$ whose magnitude serves as a measure of the intensity of the external magnetic field. The above extra term adds to the gGL potential (2) when the magnetic field is on. While $h$-term only breaks the chiral symmetry to the isospin SU(2), the $\boldsymbol{b}$-term explicitly breaks several symmetries: the time reversal symmetry, the rotational symmetry, in addition to the isospin $\mathrm{SU}(2)$ symmetry which is broken down to $\mathrm{U}_{\mathrm{Q}}(1)$.

Once we assume $\pi_{1}=\pi_{2}=0$, and take the complex notation for the condensate $\Delta=\sigma+i \pi_{3}$, the gGL potential density can be cast into the more intuitive form

$$
\begin{aligned}
\omega(\mathbf{x})= & -\boldsymbol{b} \cdot \operatorname{Im}\left[\Delta^{*} \boldsymbol{\nabla} \Delta\right]-h \operatorname{Re}[\Delta] \\
& +\frac{\alpha_{2}}{2}|\Delta|^{2}+\frac{\alpha_{4}}{4}\left(|\Delta|^{4}+|\boldsymbol{\nabla} \Delta|^{2}\right)+\frac{\alpha_{6}}{6}\left(|\Delta|^{6}+3|\Delta|^{2}|\nabla \Delta|^{2}+2\left(\operatorname{Re}\left[\Delta^{*} \boldsymbol{\nabla} \Delta\right]\right)^{2}+\frac{1}{2}\left|\nabla^{2} \Delta\right|^{2}\right) .
\end{aligned}
$$

The first two terms are the symmetry breaking sources, responsible for the current quark mass and the magnetic field, respectively. It can be easily guessed that the $h$-term favors the RKC, while the $\boldsymbol{b}$-term stabilizes the complex condensate such as the chiral spiral. We note that our $\boldsymbol{b}$-term is exactly in the same form as the one obtained in one-dimensional Gross-Neveau model [24], where it was shown that the spiral phase dominates the phase diagram. This term is forbidden in the three dimensional NJL model because it breaks the rotational symmetry. The magnetic field induces this term so that it opens the possibility that the complex condensate comes into play in the QCD phase diagram.

\section{How do magnetic fields modify the phase diagram?}

Let us first begin with the case of the chiral limit. This corresponds to ignoring the $h$-term in the gGL energy density (2). We measure every dimensionful quantity with the proper power of $\left(\alpha_{6}\right)^{-1 / 2}$. 

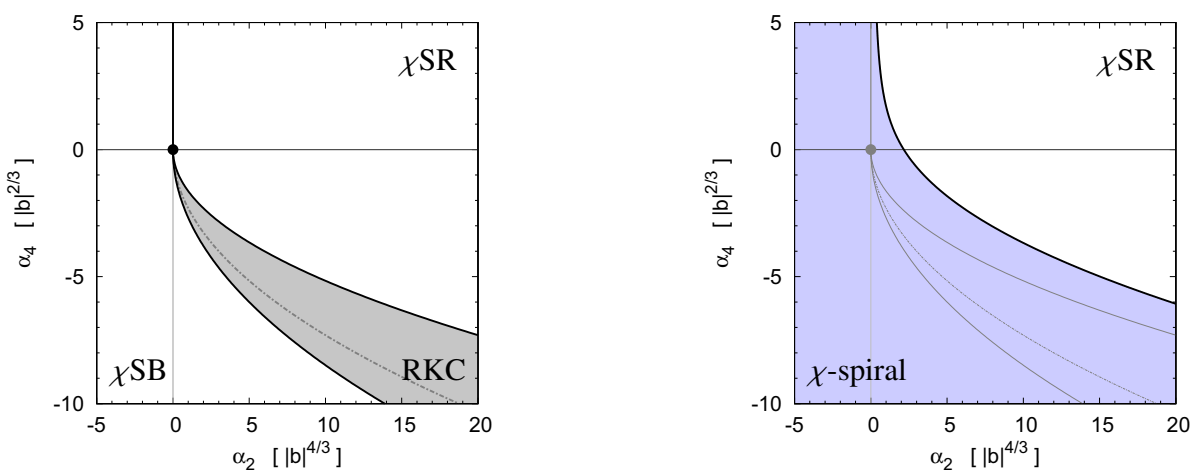

Figure 1. The phase diagrams in the chiral limit, $h=0$. Left panel: The phase diagram for zero magnetic field. Right panel: The phase diagram for nonvanishing magnetic field $\boldsymbol{b}$.

Then, we can scale out the effect of $\boldsymbol{b}$, by taking $|b|^{4 / 3}\left(|b|^{2 / 3}\right)$ for the unit of $\alpha_{2}\left(\alpha_{4}\right)$. The phase diagram for $|b|=0$ is depicted in the left panel of Fig. 1. First, note that the Lifshitz tricritical point (LTCP) is located at the origin which, in principle, has a unique map onto the $\left(\mu_{\mathrm{TCP}}, T_{\mathrm{TCP}}\right)$ in QCD phase diagram. Second, the RKC enters in between the chiral symmetric phase $(\chi \mathrm{SR})$ and the chiral symmetry broken phase ( $\chi \mathrm{SB})$. One might wonder why $|b|$ comes in the units of $\alpha_{2}$ and $\alpha_{4}$ in spite of zero magnetic field $b=0$. This is just for a convenience, and in this case $|b|$ is arbitrary. In fact, the phase boundaries are independent of $|b|$, since any critical lines are expressed by $\alpha_{4}^{2} \propto \alpha_{2}$. In the right panel, the phase diagram for nonvanishing $|b|$ is displayed. The phase structure is completely changed by the emergence of a complex chiral spiral, $\Delta(\mathbf{x})=\Delta_{0} e^{i \boldsymbol{q} \cdot \mathbf{x}}$, denoted by " $\chi$-spiral" in the figure. In this phase the direction of $\boldsymbol{q}$ is locked to the direction of the magnetic field. The LTCP is killed by the stabilization of the $\chi$-spiral phase, and there is only a second order phase transition line between $\chi \mathrm{SR}$ and $\chi$-spiral phases. We stress that this drastic change happens for an arbitrary intensity of magnetic field. It means that the standard $\chi \mathrm{SB}$ phase becomes unstable against the formation of density wave, and the LTCP will never be realized in the presence of an external magnetic field.

Next we consider the effect of current quark mass $h$ together with the magnetic field $\boldsymbol{b}$. We show in Fig. 2 the phase diagrams for four different values of magnetic fields. The phase diagram displayed in Fig. 2(a) is for $8 b=0.2 \times h^{3 / 5}$, which is the case where the effect of $\boldsymbol{b}$ is relatively weaker than the current quark mass ( $h$-term) effect. Note, however, even in this case the magnetic energy is roughly estimated as $\sqrt{e B} \sim 20 \mathrm{MeV}$ corresponding to a quite large intensity of magnetic field, $B \sim 7 \times 10^{12} \mathrm{~T}$. We see that the phase diagram is not much modified at this magnetic intensity. The magnetic field replaces only a tiny thin region near the phase boundary between the $\chi \mathrm{SR}$ and RKC phases with a modified $\chi$-spiral defined by $\Delta=M_{0}+\Delta_{0} e^{i \boldsymbol{q} \cdot \mathbf{x}}$ with $M_{0}, \boldsymbol{q}$ and $\Delta_{0}$ the variational parameters. However, a major part of the RKC and the Lifshitz critical point (LCP) itself remain intact. We conclude that the current quark mass protects the LCP and the RKC phase from a weak magnetic field. Fig. 2(b) presents the phase diagram for $8 b=1.0 \times h^{3 / 5}$, that roughly corresponds to $\sqrt{e B} \sim 40 \mathrm{MeV}\left(B \sim 3 \times 10^{13} \mathrm{~T}\right)$. At this magnetic intensity, we see a sizable region for the $\chi$-spiral. Accordingly the LCP is killed and replaced by a new critical point, where the second order transition from the $\chi$-spiral to the $\chi \mathrm{SR}$ turns into a first order one from the $\chi$-spiral to the RKC (or $\chi \mathrm{SB}$ ). Depicted in Fig. 2(c) is the phase diagram for a stronger magnetic field $8 b=5.0 \times h^{3 / 5}$, roughly, $\sqrt{e B} \sim 90 \mathrm{MeV}\left(B \sim 10^{14} \mathrm{~T}\right)$. The region for the $\chi$-spiral gets significantly magnified, and the original LCP is now completely covered by the spiral phase. There is a new critical point, denoted by a black square, where the second order 
(a)

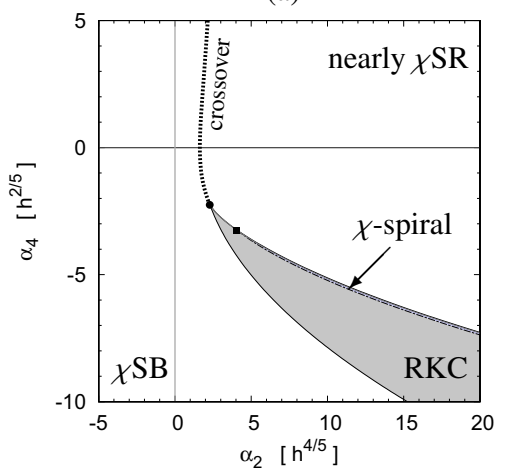

(c)

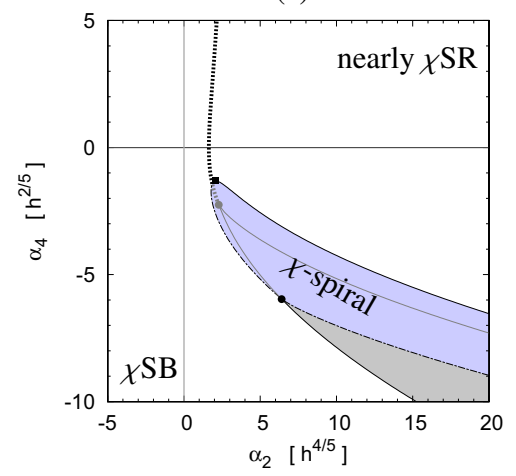

(b)

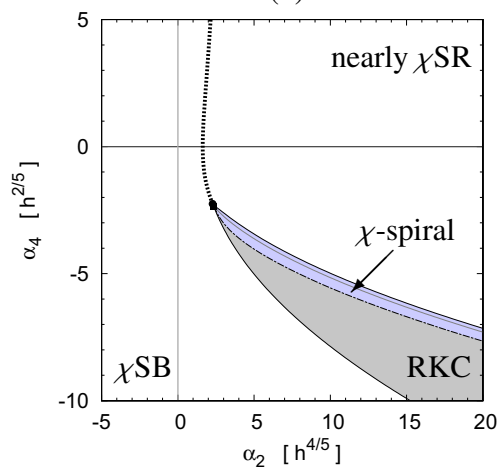

(d)

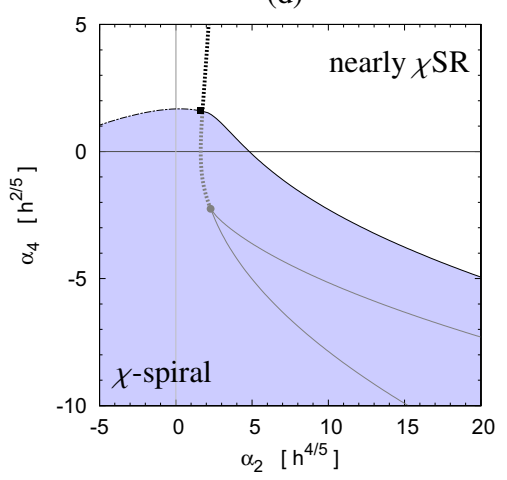

Figure 2. The phase diagrams off the chiral limit. (a): $8 b=0.2 \times h^{3 / 5}$. (b): $8 b=1.0 \times h^{3 / 5}$. (c): $8 b=5.0 \times h^{3 / 5}$. (d): $8 b=15 \times h^{3 / 5}$.

phase transition at which the $\chi$-spiral ends at high density (large $\alpha_{2}$ ) side, changes into a first order one at low density side (small $\alpha_{2}$ ). Fig. 2(d) represents the phase diagram at an even stronger magnetic field $8 b=15 \times h^{3 / 5}$, that is estimated roughly $\sqrt{e B} \sim 150 \mathrm{MeV}\left(B \sim 4 \times 10^{14} \mathrm{~T}\right)$. In this extreme case, the effect of magnetic field completely dominates over that from $h$-term. The RKC phase is replaced by the $\chi$-spiral, which now spreads over a wide region. We see that the critical point still exists on the phase boundary, where the second order phase transition turns into a first order one.

\section{Conclusions}

We studied the effects of an external magnetic field on the chiral phase structure of QCD within the generalized Ginzburg-Landau (gGL) effective action. We first derived the gGL action performing the derivative expansion up to the sixth order in condensates and spatial derivatives. Expanding the action also up to the lowest nontrivial order in a current quark mass and a magnetic field, we obtained the explicit symmetry breaking sources, $h$-term and $\boldsymbol{b}$-term, respectively. The $h$-term explicitly breaks the chiral symmetry to the diagonal isospin $\mathrm{SU}(2)$, while the $\boldsymbol{b}$-term violates the time reversal symmetry, and reduces the isospin $\mathrm{SU}(2)$ down to $\mathrm{U}_{\mathrm{Q}}(1)$, the spatial rotation symmetry $\mathrm{SO}(3)$ down to $\mathrm{O}(2)$, the rotation about the magnetic axis. It is clearly seen in the obtained gGL action that these two symmetry breaking terms have competing effects on the condensate; the former prefers the real condensate, while 
the latter favors the complex condensate spatially modulated in the direction of magnetic field. We have computed the phase diagrams for nonvanishing magnetic fields. In the chiral limit, the effect of an external magnetic field is such drastic that it completely washes out the tricritical point as well as the Real-Kink Crystal (RKC) phase. There is only a second order phase transition at which the spiral terminates. On the other hand, the effect of current quark mass was found to protect the RKC phase and the Lifshitz critical point from the erosion by a weak magnetic field. However, as the intensity of magnetic field increases, the $\chi$-spiral phase gradually invades the coast region of the high density boundary between the RKC and nearly symmetric phases. When the magnetic field strength is large enough, the effect of magnetic field prevails over that of current quark mass, and the RKC phase gets completely beaten by the chiral spiral phase. We confirmed that, in the regime of strong magnetic fields, the shape of the phase structure approaches the extreme one obtained in the chiral limit.

\section{Acknowledgements}

I thank R. Yoshiike, K. Nishiyama, and T. Tatsumi for useful discussions. I would like to express my sincere gratitude to the organizers of QCD@work 2016 at Martina Franca, especially to Pietro Colangelo and Fulvia De Fazio for their kind hospitality.

\section{References}

[1] E. Nakano and T. Tatsumi, Phys. Rev. D 71, 114006 (2005).

[2] D. Nickel, Phys. Rev. Lett. 103, 072301 (2009).

[3] For a recent review; see; M. Buballa and S. Carignano, Prog. Part. Nucl. Phys. 81, 39 (2015).

[4] R. C. Duncan and C. Thompson, Astrophys. J. 392, L9 (1992).

[5] D. E. Kharzeev, L. D. McLerran and H. J. Warringa, Nucl. Phys. A 803, 227 (2008).

[6] H. Suganuma and T. Tatsumi, Annals Phys. 208, 470 (1991).

[7] S. P. Klevansky and R. H. Lemmer, Phys. Rev. D 39, 3478 (1989).

[8] V. P. Gusynin, V. A. Miransky and I. A. Shovkovy, Phys. Rev. Lett. 73, 3499 (1994).

[9] G. S. Bali, et al., Phys. Rev. D 86, 071502 (2012).

[10] G. Endrodi, JHEP 1507, 173 (2015).

[11] F. Bruckmann, G. Endrodi and T. G. Kovacs, JHEP 1304, 112 (2013).

[12] K. Fukushima and Y. Hidaka, Phys. Rev. Lett. 110, no. 3, 031601 (2013).

[13] J. Chao, P. Chu and M. Huang, Phys. Rev. D 88, 054009 (2013).

[14] F. Preis, A. Rebhan and A. Schmitt, JHEP 1103, 033 (2011).

[15] M. Ruggieri, L. Oliva, P. Castorina, R. Gatto and V. Greco, Phys. Lett. B 734, 255 (2014).

[16] P. Costa, et al., Phys. Rev. D 92, no. 3, 036012 (2015).

[17] T. Tatsumi, K. Nishiyama and S. Karasawa, Phys. Lett. B 743, 66 (2015).

[18] G. Cao and A. Huang, Phys. Rev. D 93, no. 7, 076007 (2016).

[19] K. Nishiyama, S. Karasawa and T. Tatsumi, Phys. Rev. D 92, 036008 (2015).

[20] R. Yoshiike and T. Tatsumi, Phys. Rev. D 92, no. 11, 116009 (2015).

[21] H. Abuki, Phys. Lett. B 728, 427 (2014); Phys. Rev. D 87, no. 9, 094006 (2013).

[22] H. Abuki, D. Ishibashi and K. Suzuki, Phys. Rev. D 85, 074002 (2012).

[23] E. V. Gorbar, V. A. Miransky, I. A. Shovkovy and X. Wang, Phys. Rev. D 88, no. 2, 025043 (2013); Phys. Rev. D 88, no. 2, 025025 (2013).

[24] C. Boehmer, M. Thies and K. Urlichs, Phys. Rev. D 75, 105017 (2007). 